\title{
Physical-layer Network Coding using FSK Modulation under Frequency Offset
}

\author{
Terry Ferrett*, Hideki Ochiai ${ }^{\dagger}$, Matthew C. Valenti* \\ *West Virginia University, Morgantown, WV, USA. \\ †Yokohama National University, Yokohama, Kanagawa, Japan
}

\begin{abstract}
Physical-layer network coding is a protocol capable of increasing throughput over conventional relaying in the twoway relay channel, but is sensitive to phase and frequency offsets among transmitted signals. Modulation techniques which require no phase synchronization such as noncoherent FSK can compensate for phase offset, however, the relay receiver must still compensate for frequency offset. In this work, a softoutput noncoherent detector for the relay is derived, under the assumption that the source oscillators generating FSK tones lack frequency synchronization. The derived detector is shown through simulation to improve error rate performance over a conventional detector which does not model offset, for offset values on the order of a few hundredths of a fraction of FSK tone spacing.
\end{abstract}

\section{INTRODUCTION}

Consider the two-way relay channel (TWRC), in which two sources communicate information to one another through an intermediate relay, as the sources have no direct link. Assume that the sources utilize the same band, and transmit information frames to one another using identical modulation schemes. Using a traditional protocol, the communication occurs over four time slots - in the first two, the sources take turns transmitting information to the relay, while in the last two, the relay sequentially transmits its received information to each source.

The rate of the communication may be increased by eliminating two time steps through the physical-layer network coding protocol (PNC) [1]. The sources first transmit to the relay in the same time slot. The relay then broadcasts its received information to the sources in a single time slot. Each source detects the information transmitted by the opposite source through appropriate processing, reducing the number of communication time slots from four to two.

In this work, we consider the network configuration in which the relay performs demodulation and detection of the network-coded information using a specific modulation scheme, binary frequency-shift keying (binary FSK). FSK is a constant envelope modulation which can easily be amplified by nonlinear power amplifiers which have high power efficiency. Noncoherent detection of FSK simplifies complexity vs modulation schemes which require phase synchronization. However, FSK is not as spectrally efficient as linear modulations such as QAM, motivating the use of PNC. Previous work on this configuration has considered formulating a detector for the

M.C. Valenti's and T. Ferrett's contributions were sponsored by the National Science Foundation under Award No. CNS-0750821 and by the United States Army Research Laboratory under Contract W911NF-10-0109. network coded information bits at the relay which provides soft estimates of the network coded bits, appropriate for use with high performance channel coding techniques such as Turbo codes, under a slowly fading Rayleigh channel model [2]. The implementation of physical-layer network coding in which the relay performs demodulation and detection of the network coded bit is denoted as digital network coding.

The goal of this work is to extend the previous by relaxing the assumption that the oscillators generating FSK tone frequencies at the sources are perfectly synchronized, and develop a noncoherent relay detector which compensates for the lack of synchronization.

Crystal oscillators are commonly used to generate reference frequencies for signals such as carriers and correlator reference signals. The frequencies generated by crystal oscillators are offset from ideal specifications due to manufacturing imperfections, ambient conditions such as temperature and radiation level, as well as aging of the oscillator [3]. In this work, oscillator offsets are modeled as real values added to the frequencies of the FSK tones generated by the sources which are constant for all symbol periods, representing the case of fixed ambient conditions over a time scale short enough to neglect the effects of oscillator aging.

The effect of oscillator offset on a conventional singlesource, single-destination link has been well studied for FSK systems, comprising performance analysis, simulation, algorithms to estimate offset at the receiver, and techniques to synchronize the clocks of network nodes. In an AWGN channel, oscillator offset imposes an error-rate floor on FSK modulation when the receiver does not compensate, as shown by the performance analyses in [4] and [5]. A frequency offset estimation algorithm at the receiver may be applied to correct the offset of the received signal prior to demodulation and noncoherent detection of CPFSK, as described by [6]. Frequency offset estimates may also be used to adjust the receiver filter frequencies prior to demodulation [7]. In networks containing multiple nodes simultaneously transmitting to a single receiver, synchronization may be performed by synchronizing the clocks of all nodes to a single master [8]. In this work, our goal is to avoid the complexity of clock synchronization techniques by deriving a detection rule which compensates for frequency offset.

The specific contributions of this work are

1) A vector channel model for the received signal at the relay in the two-way relay network under digital network coding using frequency-shift keyed modulation with tone 


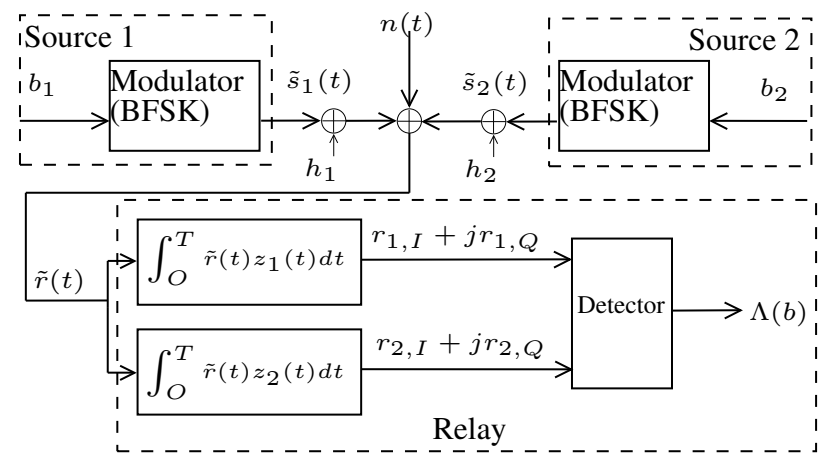

Fig. 1. Baseband Transmission Model

offsets and frequency-flat Rayleigh fading.

2) A noncoherent detector for digital network-coded FSK at the relay in the three-terminal relay network which compensates for frequency offset at the sources. The detectors exhibit reasonable performance for frequency offsets on the order of a few hundredths of the FSK tone spacing.

\section{System Model}

The two-way relay channel using the digital network coding protocol is modeled assuming binary frequency-shift keyed modulation (BFSK) and frequency-flat, slowly-varying Rayleigh fading channels, as shown in Fig. 1. During a single symbol epoch, the sources $\mathcal{N}_{i}, i \in\{1,2\}$ each generate an information bit $b_{i} \in\{0,1\}$ which is mapped to a BFSK symbol and transmitted to the relay. The signals traverse independent fading channels, corrupting the amplitude and phase of each signal. The relay receives the electromagnetic sum of the faded signals plus noise.

Each source transmits symbols having period $T$. The set of BFSK symbols at source $\mathcal{N}_{i}$ during a single symbol epoch is represented in continuous time as

$$
s_{i}(t)=\operatorname{Re}\left[\tilde{s}_{i}(t) e^{j 2 \pi f_{c} t}\right], \quad 0<t<T
$$

where $f_{c}$ is the carrier frequency, $t$ is time, and the complex baseband transmitted signal is

$$
\tilde{s}_{i}(t)=\sqrt{\frac{2}{T}} e^{j 2 \pi\left(b_{i} \Delta_{f}+d_{i}\right) t}
$$

where $b_{i}$ is the bit transmitted by source $\mathcal{N}_{i}, \Delta_{f}$ is the FSK tone frequency spacing with value $1 / T$, and $d_{i}$ is the frequency offset between source $\mathcal{N}_{i}$ and the relay.

Oscillator frequency offset at the sources is modeled as a constant, continuous value, representing an oscillator operating in static ambient conditions. Denote FSK symbols 1 and 2 as the tones generated when the information bit $b_{i}$ takes values 0 and 1 , respectively.

The signal received at the relay during a single symbol epoch is

$$
r(t)=\operatorname{Re}\left[\left(\sum_{i=1}^{2} h_{i} \tilde{s}_{i}(t)+n(t)\right) e^{j 2 \pi f_{c} t}\right]
$$

where $h_{i}=\alpha_{i} e^{j \theta_{i}}$ is the complex Gaussian channel gain between the relay and source $\mathcal{N}_{i}$ with variance $\sigma_{h}^{2}$ per complex dimension, $\alpha_{i}$ is a fading coefficient distributed as Rayleigh $(\sigma)$, and $\theta_{i}$ is the phase offset uniformly distributed as $U(0,2 \pi)$, and $n(t)$ is circularly-symmetric complex Gaussian noise. The exact values of $\alpha_{i}$ and $\theta_{i}$ are not known at the relay receiver, however, the variance of per complex dimension $\sigma_{h}^{2}$ is known. The complex received signal translated to baseband is

$$
\tilde{r}(t)=\sum_{i=1}^{2} h_{i} \tilde{s}_{i}(t)+n(t)
$$

\section{Matched Filter OUtPut AnAlysis}

In this section, the form of the signal samples at the output of the relay correlators are dervied. The received signal is translated to baseband and correlated against reference signals representing the in-phase and quadrature components of the FSK tones transmitted by the sources. One sample per symbol is assumed. The frequency of the oscillator at the relay is defined as $f_{c}$.

The samples at the output of the correlators, considering a single symbol interval, are defined as

$$
\mathbf{r}=\left[\begin{array}{l}
r_{1, I}+j r_{1, Q} \\
r_{2, I}+j r_{2, Q}
\end{array}\right]
$$

where

$$
\begin{aligned}
r_{m, I}+j r_{m, Q} & =\int_{0}^{T} \tilde{r}(t) z_{m}(t) d t, m \in\{1,2\} \\
& =\int_{0}^{T}\left[\sum_{i=1}^{2} h_{i} \tilde{s}_{i}(t)+n(t)\right] z_{m}(t) d t
\end{aligned}
$$

where $r_{m, I}$ and $r_{m, Q}$ are the in-phase and quadrature component of the $m$-th correlator sample, $\tilde{r}(t)$ is the baseband received signal, and $z_{m}(t)$ is the $m$-th correlator reference signal, defined as

$$
z_{m}(t)=\sqrt{\frac{2}{T}} e^{-j 2 \pi \Delta_{f}(m-1) t}, m \in\{1,2\}
$$

Substituting the expressions for the low-pass equivalent signals transmitted by the sources (2) and the expressions for the reference signals (7) into the expression for the correlator samples (6) and simplifying yields the final form for the correlator samples

$$
\begin{aligned}
& r_{m, I}+j r_{m, Q}= \\
& \sum_{i=1}^{2} h_{i}\left[\frac{\sin A_{i, m}}{A_{i, m}}-j \frac{\cos A_{i, m}-1}{A_{i, m}}\right]+n_{m, I}+j n_{m, Q}
\end{aligned}
$$

where

$$
A_{i, m}=2 \pi\left\{\left[b_{i}-(m-1)\right]+d_{i} / \Delta_{f}\right\}
$$

and $n_{m, I}$ and $n_{m, Q}$ are independent Gaussian random variables having mean 0 and variance $\sigma_{n}^{2}=N_{0} / 2$. 
The matched filter output statistics $r_{m, I}$ and $r_{m, Q}$ are thus Gaussian random variables having variance $N_{0} / 2$ and means which depend on the bits transmitted, the fading coefficients, and the magnitude of the frequency offsets at each source.

\section{VECTOR CHANNEL MODEL UNDER OSCILLATOR OFFSET}

This section defines a vector channel model for the signal received at the relay. It is shown that oscillator offset can be modeled as a multiplicative effect with respect to the transmitted symbol and effects of the channel. The vector notation is used to express the relay correlator samples.

Define the following vector random variable representing the bits transmitted by each source

$$
\mathbf{v}_{k}=\left[\begin{array}{ll}
b_{1} & b_{2}
\end{array}\right], \quad k \in\{1,2,3,4\}
$$

with the following mapping of events

$$
\begin{array}{ll}
\mathbf{v}_{1}=\left[\begin{array}{ll}
0 & 0
\end{array}\right] & \mathbf{v}_{3}=\left[\begin{array}{ll}
0 & 1
\end{array}\right] \\
\mathbf{v}_{2}=\left[\begin{array}{ll}
1 & 1
\end{array}\right] & \mathbf{v}_{4}=\left[\begin{array}{ll}
1 & 0
\end{array}\right]
\end{array}
$$

The bit-to-symbol mapping used by the sources is defined as follows. Denote the symbols transmitted by sources $\mathcal{N}_{1}$ and $\mathcal{N}_{2}$ as the vectors $\mathbf{s}_{1}$ and $\mathbf{s}_{2}$ respectively, with the following mapping of bits to symbols

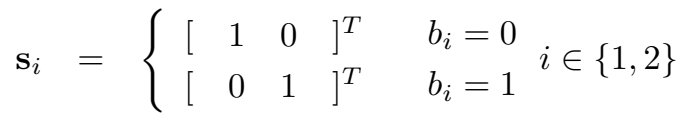

Define the following matrix containing symbols $\mathbf{s}_{1}$ and $\mathbf{s}_{2}$

$$
\mathbf{S}=\left[\begin{array}{ll}
\mathbf{s}_{1} & \mathbf{s}_{2}
\end{array}\right]
$$

with value chosen from the set of symbol matrices

$$
\begin{aligned}
& \mathbf{S}_{1}=\left[\begin{array}{ll}
1 & 1 \\
0 & 0
\end{array}\right] \mathbf{S}_{3}=\left[\begin{array}{ll}
1 & 0 \\
0 & 1
\end{array}\right] \\
& \mathbf{S}_{2}=\left[\begin{array}{ll}
0 & 0 \\
1 & 1
\end{array}\right] \mathbf{S}_{4}=\left[\begin{array}{ll}
0 & 1 \\
1 & 0
\end{array}\right]
\end{aligned}
$$

Note that in (8) the effects of oscillator offset on the correlator samples are multiplicative. This suggests that oscillator offset can be incorporated into the vector channel model as a matrix multiplication. Denote the multiplicative effect of oscillator offset at source $\mathcal{N}_{i}$ given by (8) as

$$
O_{i}\left[b_{i}, m\right]=\left[\frac{\sin A_{i, m}}{A_{i, m}}-j \frac{\cos A_{i, m}-1}{A_{i, m}}\right] i \in\{1,2\}
$$

where $A_{i, m}$ is given by (9), and the particular values of $b_{i}$ and $m$ substituted into $A_{i, m}$ are denoted by $\left[b_{i}, m\right]$.

Gathering the offset terms at source $\mathcal{N}_{i}$ as a matrix

$$
\mathbf{O}_{i}=\left[\begin{array}{cc}
O_{i}[0,1] & O_{i}[1,1] \\
O_{i}[0,2] & O_{i}[1,2]
\end{array}\right] \quad i \in\{1,2\}
$$

the channel statistics at the output of the relay demodulator considering a single symbol interval is expressed in vector form as

$$
\mathbf{r}=h_{1} \mathbf{O}_{1} \mathbf{s}_{1}+h_{2} \mathbf{O}_{2} \mathbf{s}_{2}+\mathbf{n}
$$

where $\mathbf{n}=\left[\begin{array}{ll}n_{1} & n_{2}\end{array}\right]^{T}$, and $n_{1}$ and $n_{2}$ are complex jointly Gaussian random variables.

\section{Detection Rule}

The goal of this section is to derive the optimal detection rule for the network-coded bit at the relay. A general probabilistic model of the network coding operation is developed, the detection rule is derived assuming no knowledge of channel state at the relay.

To detect the network-coded bit, the relay detector computes the log-likelihood ratio of the network-coded bit $b$

$$
\Lambda(b)=\log \frac{P(b=1)}{P(b=0)}=\log \frac{P\left(b_{1} \oplus b_{2}=1\right)}{P\left(b_{1} \oplus b_{2}=0\right)}
$$

and the log-likehood ratio of the network-coded bit given by (18) is expressed in terms of $\mathbf{v}_{i}$

$$
\begin{aligned}
\Lambda(b) & =\log \frac{P\left(\mathbf{v}_{1} \cup \mathbf{v}_{2} \mid \mathbf{r}\right)}{P\left(\mathbf{v}_{3} \cup \mathbf{v}_{4} \mid \mathbf{r}\right)} \\
& =\log \frac{P\left(\mathbf{S}=\mathbf{S}_{1} \mid \mathbf{r}\right)+P\left(\mathbf{S}=\mathbf{S}_{2} \mid \mathbf{r}\right)}{P\left(\mathbf{S}=\mathbf{S}_{3} \mid \mathbf{r}\right)+P\left(\mathbf{S}=\mathbf{S}_{4} \mid \mathbf{r}\right)} \\
& =\log \frac{p\left(\mathbf{r} \mid \mathbf{S}=\mathbf{S}_{1}\right)+p\left(\mathbf{r} \mid \mathbf{S}=\mathbf{S}_{2}\right)}{p\left(\mathbf{r} \mid \mathbf{S}=\mathbf{S}_{3}\right)+p\left(\mathbf{r} \mid \mathbf{S}=\mathbf{S}_{4}\right)}
\end{aligned}
$$

where the second line follows from noting that the symbol pairs $\mathbf{S}_{i}$ are mutually exclusive, and the third line follows from applying Bayes' rule to $P\left(\mathbf{S}_{i} \mid \mathbf{r}\right)$ and assuming that the source bits $b_{1}$ and $b_{2}$ are independent, and distributed equally likely.

Consider transmission of symbol pair $\mathbf{S}$ by the sources. The received signal at the relay may be written as

$$
\mathbf{r}=\left[\begin{array}{c}
h_{1} O_{1}\left[b_{1}, 1\right]+h_{2} O_{2}\left[b_{2}, 1\right]+n_{1} \\
h_{1} O_{1}\left[b_{1}, 2\right]+h_{2} O_{2}\left[b_{2}, 2\right]+n_{2}
\end{array}\right]
$$

where $O_{1}\left[b_{1}, 1\right], O_{2}\left[b_{2}, 1\right] . O_{1}\left[b_{1}, 2\right] . O_{2}\left[b_{2}, 2\right]$ are elements of the offset matrices in (16) selected according to the symbol pair transmitted by the sources.

Let $O_{i}\left[b_{i}, m\right]=\beta_{i, m}+\gamma_{i, m}$. Express the fading coefficients as $h_{1}=h_{1, I}+j h_{1, Q}$ and $h_{2}=h_{2, I}+j h_{2, Q}$, where $h_{1, I}, h_{1, Q}, h_{2, I}, h_{2, Q}$ are i.i.d. $\mathcal{N}\left(0, \sigma_{h}^{2}\right)$. Substituting the above definitions into (20) and simplifying,

$\mathbf{r}=\left[\begin{array}{c}\beta_{1,1} h_{1, I}-\gamma_{1,1} h_{1, Q}+\beta_{2,1} h_{2, I}-\gamma_{2,1} h_{2, Q}+n_{1, I} \\ +j\left(\gamma_{1,1} h_{1, I}-\beta_{1,1} h_{1, Q}+\gamma_{2,1} h_{2, I}-\beta_{2,1} h_{2, Q}+n_{1, Q}\right) \\ \beta_{1,2} h_{1, I}-\gamma_{1,2} h_{1, Q}+\beta_{2,2} h_{2, I}-\gamma_{2,2} h_{2, Q}+n_{2, I} \\ +j\left(\gamma_{1,2} h_{1, I}-\beta_{1,2} h_{1, Q}+\gamma_{2,2} h_{2, I}-\beta_{2,2} h_{2, Q}+n_{2, Q}\right)\end{array}\right]$

where the additive noise term $\mathbf{n}$ has been expressed in in-phase and quadrature form as $\mathbf{n}=\left[n_{1, I}+j n_{1, Q} n_{2, I}+j n_{2, Q}\right]^{T}$.

The components of the demodulator outputs are formed from the sum of scaled, independent, zero-mean complex Gaussian random variables, thus, the components are complex jointly Gaussian random variables, and completely described by their mean vector and covariance matrix. The covariance matrix assuming transmission of symbol pair $\mathbf{S}=\mathbf{S}_{i}$ is given by

$$
\mathbf{K}=E\left[\mathbf{r r}^{H}\right]
$$




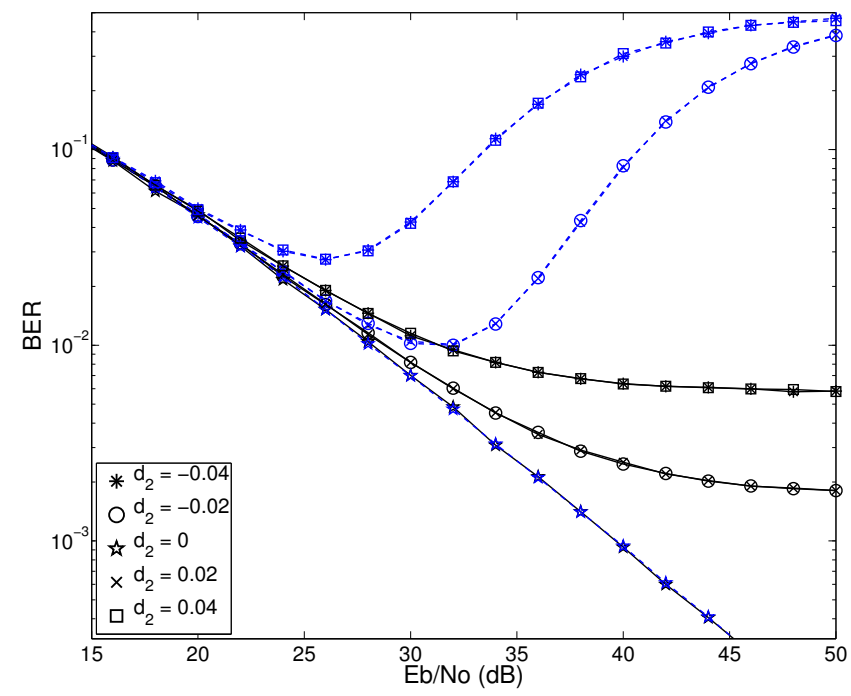

Fig. 2. Simulated performance of noncoherent detection rules under oscillator offset. Blue, dashed lines denote the detection rule which does not model offset, while black, solid lines denote the detection rule which does model offset. Offset $d_{1}=0$ for all cases.

The $\{l, k\}$-th element of $\mathbf{K}_{i}$ is given by

$$
K_{l, k}=E\left[r_{l} r_{k}\right] l, k \in\{1,2\}
$$

Enumerating all values of $K_{l, k}$

$$
\begin{aligned}
& K_{1,1}=2\left[\left(\beta_{1,1}^{2}+\gamma_{1,1}^{2}+\beta_{2,1}^{2}+\gamma_{2,1}^{2}\right) \sigma_{h}^{2}+\sigma_{n}^{2}\right] \\
& K_{1,2}=2\left(\beta_{1,1} \beta_{1,2}+\gamma_{1,1} \gamma_{1,2}+\beta_{2,1} \beta_{2,2}+\gamma_{2,1} \gamma_{2,2}\right) \sigma_{h}^{2} \\
& K_{2,1}=2\left(\beta_{1,1} \beta_{1,2}+\gamma_{1,1} \gamma_{1,2}+\beta_{2,1} \beta_{2,2}+\gamma_{2,1} \gamma_{2,2}\right) \sigma_{h}^{2} \\
& K_{2,2}=2\left[\left(\beta_{1,2}^{2}+\gamma_{1,2}^{2}+\beta_{2,2}^{2}+\gamma_{2,2}^{2}\right) \sigma_{h}^{2}+\sigma_{n}^{2}\right]
\end{aligned}
$$
by

The distribution of the demodulator outputs is thus given

$$
p\left(\mathbf{r} \mid \mathbf{S}_{i}\right)=\frac{1}{(2 \pi)^{2}|\mathbf{K}|} \exp \left(-\mathbf{r}^{H} \mathbf{K}^{-1} \mathbf{r}\right)
$$

where $\mathbf{K}^{-1}$ is the inverse of the covariance matrix $\mathbf{K}$, and $|\mathbf{K}|$ is the determinant. The log-likelihood ratio of the networkcoded bit $b$ under noncoherent operation is computed by substituting the conditional distribution (25) into the general expression for the log-likelihood ratio (19), where

$$
\log \left[p\left(\mathbf{r} \mid \mathbf{S}=\mathbf{S}_{i}\right)\right]=-2 \log 2 \pi-\log |\mathbf{K}|-\mathbf{r}^{H} \mathbf{K}^{-1} \mathbf{r}
$$

is the logarithm of (25).

\section{Simulation Study}

This section presents the simulated error rate performance of the noncoherent detector presented in Section V. For all simulations, the frequency offset is normalized with respect to the tone spacing $\Delta f$. Assume that the symbol rate transmitted by the sources is proportional to tone spacing. The simulated frequency offset values are several hundredths of a tone spacing, representing the case in which offset is a modest fraction of symbol rate. Error rate performance is given for several values of frequency offset at the sources with and without

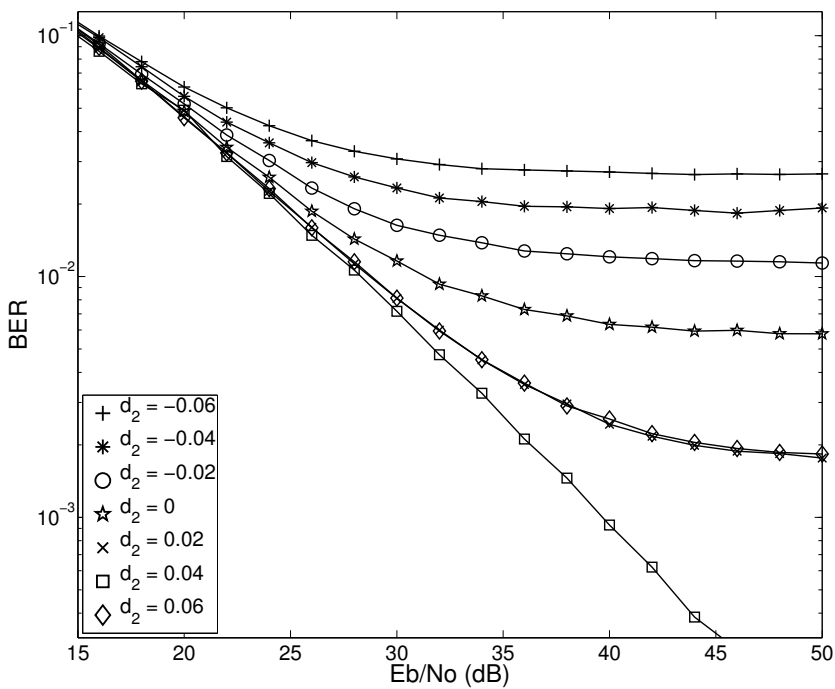

Fig. 3. Simulated performance of noncoherent detection rule incorporating frequency offset assuming nonzero offsets at both sources. Offset $d_{1}=0.04$ for all cases.

an error-correcting channel code. The channel code used in simulation is the UMTS Turbo code [9]. A full description of the application of Turbo codes to the network considered in this work is given in [2]. Performance of the detector which incorporates oscillator offset knowledge is compared against the receiver rule which does not explicitly model offset.

The error rate performance considering offset between the relay and a single source is shown in Fig. 2. This scenario models the case in which the oscillator at the relay is frequency locked to the oscillator at source 1 . The detection rule that does not incorporate offset reaches a minimum error rate and then degrades in performance as a function of SNR. The detection rule incorporating offset outperforms the rule which does not, however, the error rate encounters an error floor as SNR increases. Further analysis is required to determine the reason that the detection rule incorporating offset reaches an error floor. The implication of the simulation results is that offsets less than 0.04 fractions of a tone spacing permit error rates lower than $10^{-2}$.

The performance of the noncoherent detection rule under an offset between both sources and the relay is shown in Fig. 3 . This scenario models the case in which the oscillator frequency at the relay is not locked to either source. The numerical results imply that performance is dominated by the relative offset between the oscillators at source 1 and 2, as performance in the case of $d_{1}=d_{2}=0.04$ is approximately equal to the case of $d_{1}=d_{2}=0$ shown in Fig. 2. Absolute values of offset lead to an energy loss which is insignificant in the offset values considered in simulation.

Performance of the noncoherent detection rule when a Turbo code is applied to protect the information bits at both sources is shown in Fig. 4. The rate of the Turbo code is chosen to illustrate that the detection rule which models offset is capable of achieving lower error rates for particular values of SNR than 


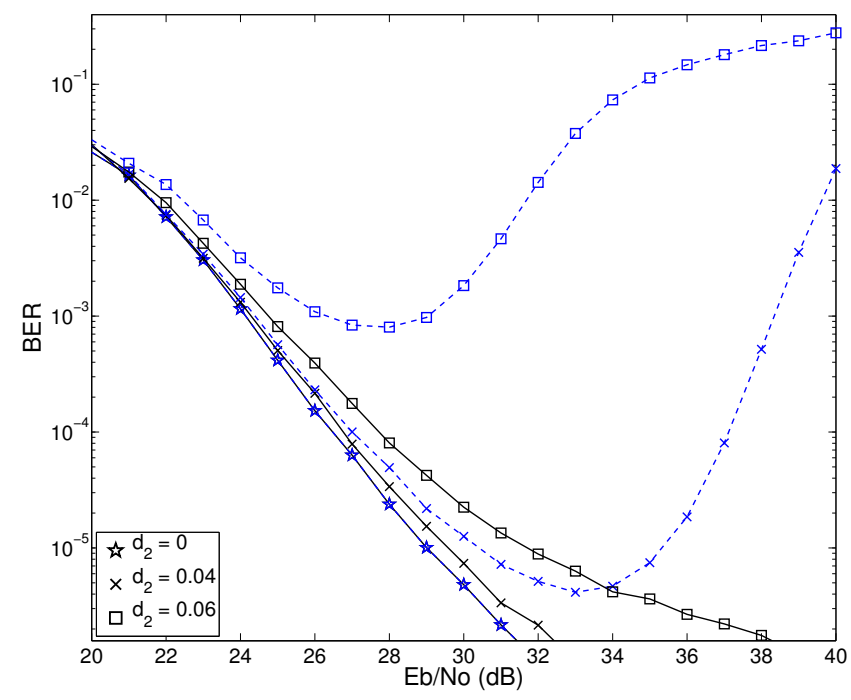

Fig. 4. Simulated performance of noncoherent detection rule incorporating frequency offset assuming nonzero offsets at both sources. Offset $d_{1}=0$ for all cases. A rate $4500 / 6500$ Turbo code is applied to all simulations. Blue, dashed lines denote the detection rule which does not model offset, while black, solid lines denote the detection rule which does model offset.

the rule which does not model offset. Consider the simulation case of $d_{2}=0.06$. At an SNR of $36 \mathrm{~dB}$, the rule which models offset achieves reaches an error rate below $10^{-5}$, while the rule which does not exhibits an error rate higher than $10^{-1}$.

Examples of radio parameters exhibiting frequency offsets on the order of hundredths of a tone spacing are shown in Table I. Frequency offset for actual crystal oscillators is typically a function of the particular carrier frequency at which the oscillator is driven. A tutorial on the specification of offset for actual oscillators is [3]. The tabulated values of are representitive of the capabilities of contemporary platforms, such as the Universal Software Radio Peripheral (USRP) [10].

\section{CONCLUSION}

A noncoherent detector for the relay in the two-way relay channel using the digital network coding protocol is developed. The detector is capable of compensating for oscillator frequency offsets, achieving significant performance improvement over a detection rule which does not consider offset. The detector produces soft outputs appropriate for use with soft-decision decoding algorithms.

When the relay is capable of locking its oscillator frequency to one of the sources, the noncoherent detector is capable of achieving uncoded error rates below $10^{-2}$ for frequency offset values at the opposite source on the order of a few hundredths of a fraction of FSK tone spacing. Appropriate Turbo code rates at the sources allows the detector which models offset to achieve lower error rates than the detector which does not. As SNR tends to infinity, the error performance of the detector tends to an error floor. Further analysis is required to determine the source of this floor. When the relay is not capable of locking its oscillator frequency to either source, performance is dominated by the difference between the offsets at the sources.

\section{REFERENCES}

[1] S. Zhang, S. C. Liew, and P. P. Lam, "Physical-layer network coding," in Proc. MobiComm, pp. 358-365, 2006.

[2] M. C. Valenti, D. Torrieri, and T. Ferrett, "Noncoherent physical-layer network coding with FSK modulation: Relay receiver design issues," IEEE Trans. Commun., Sept. 2011.

[3] J. R. Vig, "Introduction to quartz frequency standards," Electronics and Power Sources Directorate, pp. SLCET-TR-92-1 (Rev. 1), Oct. 1992. [Online]. Available: http://www.ieee-uffc.org/frequency_control/ teaching.asp? name $=$ vigtoc

[4] S. Hussain, S. Barton, and S. Shepherd, "Non-coherent detection of fsk signals in the presence of oscillator phase noise in an awgn channel." IEEE Trans. Veh. Technol., pp. 1552-1556, 1994.

[5] H.-G. Ryu, Y. Li, and J.-S. Park, "Effects of frequency instability caused by phase noise on the performance of the fast fh communication system," IEEE Trans. Veh. Technol., pp. 1626-1632, Sept. 2004.

[6] G. Caire and C. Elia, "A new symbol timing and carrier frequency offset estimation algorithm for noncoherent orthogonal M-CPFSK," IEEE Trans. Commun., pp. 1314-1326, Oct. 1997.

[7] Y. Huang, K. Fan, and C. Huang., "A fully digital noncoherent and coherent GMSK receiver architecture with joint symbol timing error and frequency offset estimation," IEEE Trans. Veh. Technol., pp. 863-874, May 2000.

[8] Y.-S. Tu and G. J. Pottie, "Coherent cooperative transmission from multiple adjacent antennas to a distant stationary antenna through awgn channels," IEEE Trans. Veh. Technol., pp. 130-134, Aug. 2002.

[9] European Telecommunications Standards Institute, "Universal mobile telecommunications system (UMTS): Multiplexing and channel coding (FDD)," 3GPP TS 25.212 version 3.4.0, Sept. 232000.

[10] M. Ettus, "Tx and rx daughterboards for the usrp tm software radio system," www.ettus.com, 2011. [Online]. Available: http://www.ettus. com/downloads/ettus_daughterboards.pdf
TABLE I

EXAMPLE VALUES OF OSCILLATOR OFFSET

\begin{tabular}{|c|c|c|c|}
\hline $\begin{array}{c}\text { Carrier } \\
\begin{array}{c}\text { Frequency } \\
(\mathrm{GHz})\end{array}\end{array}$ & $\begin{array}{c}\text { Tone } \\
\text { Spacing, } \Delta f \\
(\mathrm{kHz})\end{array}$ & $\begin{array}{c}\text { Offset, } d \\
(\mathrm{kHz})\end{array}$ & $\begin{array}{c}\text { Offset, } \frac{d}{\Delta f} \\
\text { (normalized) }\end{array}$ \\
\hline 1.0 & 250 & 10 & 0.02 \\
\hline 2.4 & 2400 & 48 & 0.04 \\
\hline 4.9 & 3200 & 98 & 0.03 \\
\hline
\end{tabular}

